\title{
Incidentally Detected Coronary Artery Fistula Detected on Routine Perioperative Transesophageal Echocardiography
}

S Subash, BM Shivaprasad, A Jayant, Goverdhan Dutt Puri

\begin{abstract}
Coronary artery fistulae (CAF) are rare anomalies. Herein, we describe the use of intraoperative transesophageal echocardiography (TEE) in the diagnosis and management of CAF. A 3-year-old child diagnosed to have ostium secundum atrial septal defect (OS ASD) was posted for surgical closure under cardiopulmonary bypass (CPB). Post procedure TEE revealed a single continuous turbulent jet toward right atrium near the lateral wall of tricuspid region and was diagnosed as CAF communicating right coronary artery and right atrium. Surgical ligation of the CAF under CPB was done and the child was discharged postoperatively without complications. This case highlights the utility of intraoperative TEE in the diagnosis and management of CAF.
\end{abstract}

Keywords: Coronary artery fistula, Coronary cameral fistula, Coronary arterial fistula, Coronary anomaly, Transesophageal echocardiography.

How to cite this article: Subash S, Shivaprasad BM, J ayant A, Puri GD. Incidentally Detected Coronary Artery F istula Detected on R outine Perioperative Transesophageal E chocardiography. J Perioper Echocardiogr 2013;1(1):27-29.

\section{Source of support $\mathrm{Nil}$}

Conflict of interest: None declared

\section{CASE REPORT}

A 3-year-old child was diagnosed in early infancy to have ostium secundum atrial septal defect (OS ASD), and small perimembranous ventricular septal defect (V SD) and patent ductus arteriosus (PDA). On echocardiographic follow-up both the PDA and VSD were found to have closed spontaneously before the child attained 1 year of age. She presented to the hospital again with a history of recurrent chest infections and poor weight gain. Clinical examination revealed a systolic murmur most prominent in the pulmonic area and a wide split second heart sound. Preoperative chest skiagram showed cardiomegaly with computed tomographic (CT) ratio 0.55 , electrocardiography showed normal sinus rhythm of heart rate at 100 beats/minute, normal axis and right bundle branch block. The biochemical, coagulation and hematological investigations were within normal limits. A preoperative transthoracic echocardiogram showed a single $23 \mathrm{~mm}$ OS ASD, right heart enlargement; right ventricular systolic pressure was estimated to be $37 \mathrm{~mm}$ $\mathrm{Hg}+$ right atrial mean pressure with normal biventricular function. No other anomalies were noted and cardiac catheterization was not deemed necessary. The patient was posted for surgical closure of the A SD under cardiopul monary bypass (CPB).

Following standard anesthesia induction a 3 to $7 \mathrm{M} \mathrm{Hz}$ pediatric transesophageal echo probe (S7-3t, Philips IE 33 ultrasound system, B othell, W A) was inserted uneventfully. The surgery was performed through a median sternotomy. Even as the preoperative findings were being confirmed on transesophageal echo the child developed a spell of hemodynamically significant supraventricular tachycardia. The surgeon chose to immediately proceed with institution of CPB and a full TEE examination was not done in view of the preoperative diagnosis of isolated ASD. The defect was closed with Dacron patch and initial separation from bypass was without event. Postprocedure TEE revealed no residual defect but instead revealed an unusual turbulence on color Doppler interrogation in the pretricuspid region; this was sited in relation to the posterolateral right atrial free wall just above the tricuspid annulus (Fig. 1). The video (V ideo 1, Fig. 2) demonstrated continuous flow throughout the cardiac cycle as would be expected in a fistulous arteriovenous communication. Since, there was a suspicion now of a high velocity cameral fistula the coronaries were visualized carefully and the right coronary artery was found to be slightly bigger or nearly as big as its left-sided counterpart (Fig. 3). In confirmation, slightly occluding the right coronary artery in its proximal course would diminish the turbulent jet. CPB was reinstituted, right atrium was opened and the fistula tract opening into right atrium was confirmed, atrial end of this fistula was closed from within the right atrium. The patient was easily separated from CPB subsequently and had an uneventful postoperative course.

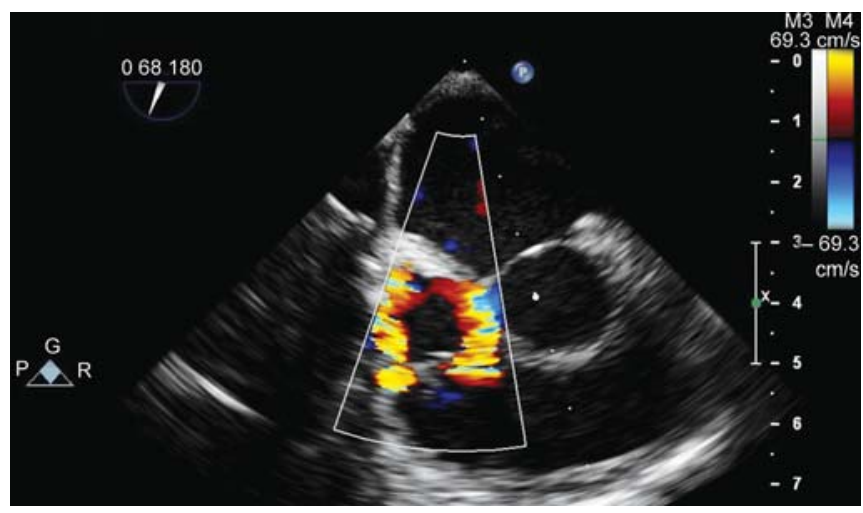

Fig. 1: Midesophageal short axis view showing turbulent jet in tricuspid region 


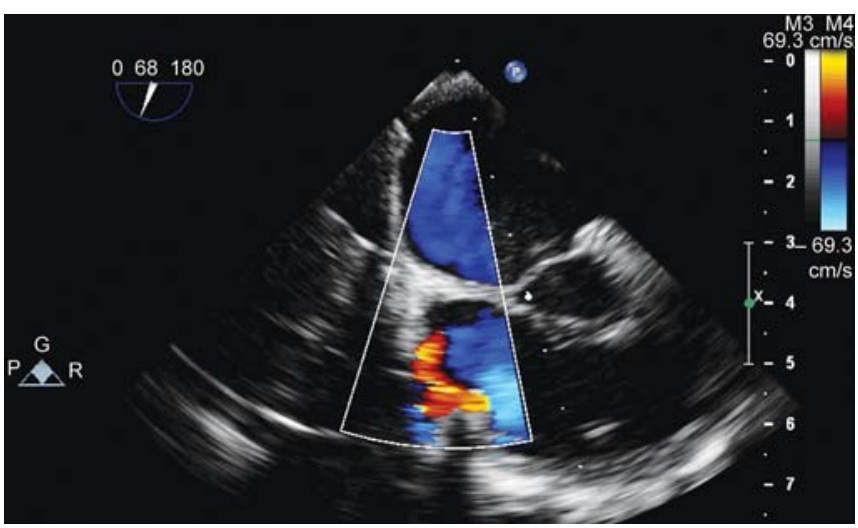

Fig. 2: Midesophageal aortic short axis view showing turbulent jet in diastolic phase

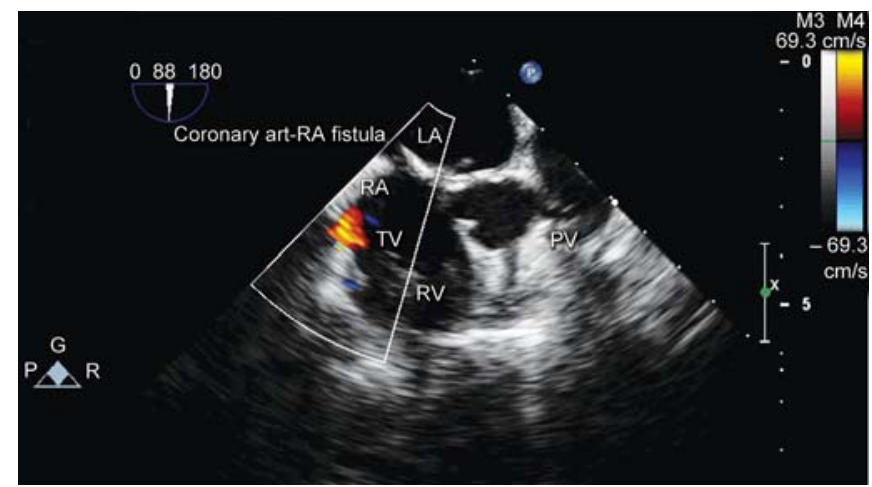

Fig. 3: Midesophageal aortic short axis view showing dilated right coronary artery

\section{DISCUSSION}

Coronary artery fistulae (CA F) are reported with a frequency of 0.1 to $0.2 \%$ during routine coronary angiography. ${ }^{1}$ They account for about half of all the congenital anomalies in coronary anatomy. ${ }^{2} \mathrm{~A}$ congenital coronary atriovenous (A V) fistula was first described by $\mathrm{K}$ rause in $1865 .{ }^{3}$ Swan et al were the first to repair coronary AV fistula using CPB in 1959. ${ }^{4}$

Coronary A V fistula most commonly originate from the right coronary artery, around 35\% originate from the left coronary and only $5 \%$ arisefrom both of these vessels. M ore than $90 \%$ of fistulae drain into the right heart. The surgical objective is closure of the fistulous tract without compromising the normal coronary circulation.

With regard to the imaging of these anomalies exact delineation may almost always need selective coronary angiography. ${ }^{5}$ However, echocardiography equally provides clues that can then subsequently be delineated by angiography. The most obvious of these is in the form of a dilated proximal culprit coronary artery; with close relation to the transesophageal echocardiography (TEE) probe, the proximal origins of the coronary arteries are exquisitely delineated by this modality. ${ }^{6}$ Occasionally the actual fistulous tract is imaged, ${ }^{7}$ more often however, the distal cameral end is alone visualized on color Doppler interrogation as in our case which demonstrates diastolic blood flow into the chamber. Importantly chamber dilation is unusual in this kind of shunt lesion. ${ }^{5}$ The other possible causes of this finding may be rupture of sinus of $V$ alsalva (RSOV) in to right atrium, ${ }^{8}$ tricuspid valve leaflet perforation ${ }^{9}$ and Gerbode shunt. ${ }^{10} \mathrm{H}$ ow ever, in RSOV into right atrium and Gerbode shunt the turbulent flow are mostly seen arising from left ventricular outflow tract into right atrium. In case of tricuspid valve leaflet perforation as well as Gerbode shunts the regurgitation jet will not have continuous flow. In our case the site of the turbulent jet, anatomically widely apart from the left ventricular outflow tracts and the continuous flow of this jet helped in ruling out the other above mentioned possibilities. A fter ligation TEE can prove useful in delineating completeness of ligation and al so to document any new onset regional or ventricular ischemic dysfunction that might warrant surgical revision of the ligation procedure. ${ }^{11}$ In conclusion, TEE is a useful adjunct in the diagnosis and management of CA F.

\section{REFERENCES}

1. Gillebert C, V an Hoof R, V an de W erf $F$, et al. Coronary artery fistulas in an adult population. Em Heart J 1986;7:437-43.

2. L uo L, K ebede S, W u S, Stouffer GA . Coronary artery fistulae. A m J Med Sci 2006;332:79-84.

3. K rause $W$. Ueber den U rsprung einer accessorischen $A$. coronaria cordis aus der A . pulmonalis. Z Ratl M ed 1865;24:225.

4. Swan H, Wilson JN, Woodwark G, Blount SG J r. Surgical obliteration of a coronary artery fistula to right ventricle. A M A A rch Surg 1959;79:820.

5. Herlong RJ. Congenital anomalies of the coronary arteries. In Lai WM, Mertens LL, Cohen MS, Geva T, (Eds). Echocardiography in pediatric and congenital heart disease. Chicester: Wiley-Blackwell 2009;502-22.

6. Giannocarro PJ, Sochowski RA, Morton BC, Chan KL. Complementary role of transesophageal echocardiography to coronary angiography in the detection of coronary artery anomalies. Br Heart J 1993;70:70-74.

7. Chung P, Huang P, Liu Y . Transesophageal echocardiography, more than a diagnostic tool: U se during surgical ligation of coronary artery fistulae. J Cardiothorac Surg 2012;7:28.

8. Chang $\mathrm{CC}, \mathrm{Chin} \mathrm{CH}, \mathrm{C}$ hen $\mathrm{ML}$, et al. Sinus of $\mathrm{V}$ alsalva aneurysm with rupturing into the right atrium: $\mathrm{A}$ case report and review of the literature. A cta Cardiol Sin 2006;22:96-101.

9. Badano LP, A gricola $E$, Perez de Isla $L$, et al. Evaluation of the tricuspid valve morphology and function by transthoracic realtime three-dimensional echocardiography. Eur J Echocardiogr 2009;10:477-84.

10. Velebit V, Schoneberger A, Ciaroni S, et al. A cquired left ventricular-to-right atrial shunt (Gerbode defect) after bacterial endocarditis. Tex Heart Inst J 1995;22:100-02.

11. L owson SM, Danner JH, Dent J M, Rich GF. Transesophageal echocardiography in the diagnosis and management of a coronary artery fistula. A nesth A nalg 1996;83:1117-19. 
Incidentally Detected Coronary Artery Fistula Detected on Routine Perioperative Transesophageal Echocardiography

\section{ABOUT THE AUTHORS}

\section{S Subash}

Senior Resident, D epartment of A nesthesia and IntensiveCare, Postgraduate Institute of M edical Education and Research, Chandigarh, India

\section{BM Shivaprasad}

A ssistant Professor, Department of Cardiothoracic and V ascular Surgery (CTVS), Postgraduate Institute of M edical Education and Research, Chandigarh, India

\section{A Jayant}

A ssistant Professor, Department of Anesthesia and Intensive Care Postgraduate Institute of M edical Education and Research, Chandigarh India

\section{Goverdhan Dutt Puri (Corresponding Author)}

Professor, D epartment of A nesthesia and Intensive Care, Postgraduate Institute of M edical Education and Research, Chandigarh, India e-mail: gdpuri007@hotmail.com 